\title{
A MODEL STUDY OF ISOVOLUMIC AND NON-ISOVOLUMIC LEFT VENTRICULAR CONTRACTIONS*†
}

\author{
J. H. J. M. VAN DEN Broek and J. J. DenIER VAN DER GoN \\ Department of Medical and Physiological Physics, University of Utrecht, \\ Sorbonnelaan 4, Utrecht, The Netherlands
}

\begin{abstract}
A model of the left ventricle is proposed. It consists of a thick-walled cylinder-like cavity, the wall of which contains muscle fibres. Orientation and length of the fibres are functions of their position in the wall. During changes of preload and during ejection the dimensions of the cylinder change in a prescribed way. A sliding filament model in series with a real or apparent elastic element is appliod to simulate wall tension from which the pressure in the ventricle results. To test the model and to fit the relevant parameters, experiments on isolated perfused rabbit hearts were carried out. The model could simulate isovolumic and nonisovolumic contractions reasonably well in the case when a series elasticity allowing about $6 \%$ fibre shortening was present and a preload dependent activation function was applied. Finally it must be concluded that for an accurate estimation of the parameters, not only pressure development and flow must be recorded, but also certain overall ventricular dimensions.
\end{abstract}

\section{INTRODUCTION}

In the literature, muscle models often relate contraction processes to muscle force and heart or ventricular models relate muscle force or ventricular wall stress to developed pressure. Streeter et al. (1970) used a thick-walled ellipsoidal ventricular model in which fibre stress is calculated from static force-length data derived from papillary muscle experiments. The effect of sarcomere shortening velocity on the developed force is not taken into account. Wong (1973) also used a thick walled ellipsoidal ventricular model. The muscle fibres are thought to contain a contractile element and series and parallel elastic properties. The behaviour of the contractile element is based on Huxley's muscle contraction theory (Huxley, 1957). The model can be used to calculate the principal stresses and ventricular pressure during isovolumic (isometric) contractions.

In this study a model is presented which applies to isovolumic as well as non-isovolumic contractions. The aim is to test some current theories concerning physiological processes of cardiac contraction and to investigate which parameters may be estimated with reasonable accuracy from measurable quantities such as ventricular pressure and flow. The shape of the ventricle is approximated by a thick-walled cylinder. The wall of the cylinder consists of muscle fibres. The orientation and length of these fibres will have different values at different wall layers. The model offers the opportunity to study in a fairly simple way the

* Received 14 September 1978 ; in revised form 12 February 1979.

+ Some of the results of this study were communicated at the Third International Conference on Cardiovascular System Dynamics, Leiden, The Netherlands, August 1978. influence of a number of relevant features connected to ventricular geometry and muscle contraction. This model is an improvement on spherical models (Back, 1977), especially if one considers the equatorial region of the ventricle. Besides, in a cylinder model, muscle fibre orientation may be incorporated in a more significant way. A better overall approximation may certainly be obtained using ellipsoidal models. However, analysis and computations are much more elaborate while the essential mechanisms would be obscured. Furthermore, the approximation of the equatorial region of the ventricle to a cylinder is not much worse than using an ellipsoid, and in any case preliminary investigations have shown that the final results and conclusions of this study appear to be valid for both types of model (Van den Broek and Denier van der Gon, 1979).

The muscle model used consists of a contractile element obeying Huxley's sliding filament theory, and a series elastic element. It is challenged nowadays if any series elasticity outside the cross-bridges is present in muscle fibres (Huxley and Simmonds, 1971; Pollack and Krueger, 1976). However, even if no real series elastic element does exist, an apparent series elastic behaviour will still be present due to bending of the valves during contraction, compression of the coronary arteries, torsion, etc. As a result, sliding of muscie filaments will occur even if the contraction is isovolumic. It may be that a series elastic behaviour of the ventricle has to be interpreted in this way.

We will confine ourselves to the active phase of the contraction. For this reason, parallel elasticity, describing the elastic properties of the non-activated muscle is not considered here, as its contribution to the elastic behaviour is very small during the greater part of the active phase (Schiereck and Boom, 1978). It can, however, be easily incorporated in the model. But such 
an extension only makes sense if reliable passive pressure-volume relations of the ventricle are known.

Some experimental results will be used to test the validity and usefulness of the developed model.

\section{MODEL}

\section{Muscle model}

According to Huxley's theory (1957), force generation by the sarcomeres in response to activation results from chemical interactions, i.e. the so-called cross-bridges. $n$ will denote the cross-bridge density (number of cross-bridges per unit of cross-bridge length in one half-sarcomere) and $N$ the density of myosin heads (per half-sarcomere) which may form cross-bridges. $n$ may change with time due to coupling of not yet coupled bridges $(N-n)$ with a rate constant $f$, and due to uncoupling of coupled bridges $(n)$ with a rate constant $g$

$$
\begin{aligned}
\frac{\mathrm{D} n(x, t)}{\mathrm{D} t}=f(s, x, t)[N(s, x, t) & -n(x, t)] \\
& -g(s, x, t) n(x, t),
\end{aligned}
$$

where $t$ is time, $x$ cross-bridge length, $s$ sarcomere length. $\mathrm{D} / \mathrm{D} t$ is the so called material derivative, i.e. the time derivative with respect to a frame moving with the cross-bridge density distribution. Conversion to a fixed frame is possible with help of the well known formula from hydrodynamics

$$
\frac{\mathrm{D}}{\mathrm{D} t}=\frac{\partial}{\partial t}+v_{s} \frac{\partial}{\partial x}
$$

$\partial / \partial t$ is the so called local derivative. $v_{s}$ is the sarcomere shortening velocity (per half-sarcomere), given by $v_{s}=$ $\mathrm{d} x / \mathrm{d} t$.

In general $v_{s}$ is determined by the developed force and the load of the muscle, $v_{s}=v_{s}(n, t) . N$ is supposed to be independent of $x$ and $t$. $N$ is still a function of sarcomere length or preload: $N=N(s)$. The function chosen is similar to the force length relation as measured by Pollack and Krueger (1976). The resulting partial differential equation

$$
\begin{aligned}
& \frac{\partial n(x, t)}{\partial t}+v_{s}(n, t) \frac{\partial n(x, t)}{\partial x}= \\
& \quad f(s, x, t)[N(s)-n(x, t)]-g(s, x, t) n(x, t)
\end{aligned}
$$

is to be solved numerically. The functions $f, g$ and $v$ have to be known together with the initial and boundary conditions (see Appendix). If we suppose the cross-bridges to behave as linear elastic bonds with stiffness $Z$, the developed force is equal to

$$
F_{d}(t)=\int_{-\infty}^{+\infty} n(x, t) Z x \mathrm{~d} x .
$$

Inertial forces of the filaments may be neglected. Viscous forces within the sarcomeres can be neglected at velocities lower than 1.5 muscle length per sec (Noble, 1977). In normal hearts, velocities do not exceed this value. The only load on the half sarcomere with length $l_{s}$, velocity $v_{s}$ and force $F_{p}$, will be the force $F_{e}$ exerted by the series elastic element (SEE) with length $l_{\text {e }}$ and velocity $v_{*}$. The sarcomere-SEE system, called the muscle fibre, will have a length $l_{m}$, velocity $v_{m}$ and force $F_{m}$, so that

$$
l_{s}+l_{e}=l_{m} ; v_{s}+v_{e}=v_{m} ; F_{s}=F_{e}=F_{m} .
$$

The forces are developed forces; that is to say actual forces minus preload. During isometric contractions $v_{m}=0$. The sarcomeres shorten at the expense of the real or apparent SEE's (see Introduction). The dimensionless force-length relation of the SEE will be described by a more often used exponential relation :

$$
F_{e} / F_{\max }=F_{0}\left(\mathrm{e}^{2 l_{\mathrm{e}} / l_{1,}}-1\right) \text {. }
$$

$F_{\max }$ is the maximum muscie force of a sarcomere isometric contraction at zero preload, $I_{0}$ is the muscle fibre length at zero preload, $F_{0}$ a constant and $\alpha$ a kind of stiffness. The length I. is considered to be zero if no external force is exerted by the muscle.

\section{Cross-bridge kinetics}

The formation of a cross-bridge is highly influenced by the intracellular calcium concentration $\left[\mathrm{Ca}^{++}\right]$ (Weber and Murray, 1973), which in turn depends on the time course of the action potential (Kaufmann et al., 1974). In this study we suppose $\left[\mathrm{Ca}^{++}\right]$to be equal to the rate function $f(t)$, as suggested by Wong (1971)

$$
\begin{aligned}
& t \geqslant t_{0}\left[\mathrm{Ca}^{++}\right](t)=f(t) \\
& \quad=A\left[\mathrm{e}^{-b_{1}\left(t-t_{0}\right)^{2}}-\mathrm{e}^{-b_{2}\left(t-t_{0}\right)^{2}}\right], \quad b_{2}>b_{1} \\
& t<t_{0}\left[\mathrm{Ca}^{++}\right](t)=f(t)=0 .
\end{aligned}
$$

In this pure phenomenological equation, the parameters $A, b_{1}$ and $b_{2}$ have no direct physiological meaning. They determine the shape of $f(t)$ and they have to be interpreted in relation to this shape. $t_{0}$ is the time at which the contraction starts.

The $x$-dependency of $f$ and $g$, as described by Huxley (1957), is

$$
\begin{aligned}
& x<0 \quad f=0 \quad g=g_{2} \\
& 0 \leqslant x \leqslant h \quad f=f Q(x) \quad g=g_{1} Q(x) \\
& x>h \quad f=0 \quad g=g_{1} Q(x) .
\end{aligned}
$$

$h$ is the maximum bridge length for attachment. Huxley postulated in his model $Q(x)=x / h$, which has, however, no clear physiological meaning. As our simulations suggested that the influence of such a $x$ dependency of $Q(x)$ on the resulting force is rather small, we will suppose $Q(x)=1$.

The twitch duration and maximum force vary with sarcomere length because the actin-myosin overlap changes and also because sarcomere length influences the calcium kinetics (see for recent review : Jewell, 1977). This last effect is, moreover, time dependent; it takes a certain time before the calcium processes are adapted to a new sarcomere length (Lakatta and Jewell, 1977). Until now, however, no useful models 
have been developed to describe all these influences.

In our model, the adaptation process from one experimental situation to another is not considered. The influence of actin-mysin overlap, together with the length dependent calcium kinetics on the maximum twitch force, are incorporated in the force-length relation. The shape of a twitch is infiuenced by making the activation function sarcomere length dependent. In the literature it is suggested that the time to peak tension in particular is affected by sarcomere length (Allen et al., 1974). Our experiments show an influence on the descending limb of the pressure-time curve (see Fig. $3 a$ and b). Therefore the descending limb of $f$ will depend on sarcomere length in this study. This can be achieved by varying $b_{1}$ in equation (7). The other constants $A$ and $b_{2}$ will be changed in such a way that the maximum value of $f$ $\left(f_{\max }\right)$ and the time at which this value reaches its maximum ( $f_{\max }$ ) remain constant (see Fig. 1). For simplicity, changes in $b_{1}$ will be related to volume changes of the ventricle (see next paragraph) instead of to length changes of the individual muscle fibres in the wall, since the difference between the two approaches is very small. The influence of a volume change due to a preload change will be separated from the influence of a volume change due to ejection, because this last efiect is more instantaneous. To a first approximation a linear relation is used

$$
b_{1}=b_{0}\left(1-\frac{V_{p}-V_{0}}{V_{0}} b_{p}-\frac{V-V_{p}}{V_{0}} b_{e}\right),
$$

where $b_{0}, b_{p}$ and $b_{e}$ are parameters, $V_{0}$ is the volume at a preload of $0 \mathrm{mmHg}, V_{p}$ is the volume at a higher preload and $V=V(t)$ the volume during ejection.

\section{Ventricular model}

Orientation and length of the muscle fibres in the ventricular wall are functions of their position (Spotnitz et al., 1966; Streeter et al., 1969; Yoran et al., 1973; Ross and Streeter, 1975). In our thick-walled cylinder model we will use at zero filling pressure a fibre orientation varying linearly from $+60^{\circ}$ (inner wall) to $-60^{\circ}$ (outer wall) and a half-sarcomere length $l_{m}$ of

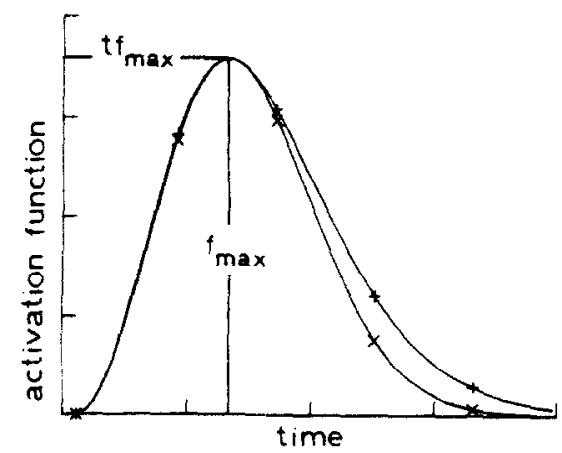

Fig. 1. Activation function $f$ as function of time for different values of $b_{1}$, under the condition that $f_{\text {max }}$ and $t f_{\text {max }}$ remain constant. In the upper curve $(+) b_{1}$ has a smaller value than in the lower curve $(x)$.
$0.86 \mu \mathrm{m}=l_{0}$. These values may be seen as approximations of measurements by Streeter et al. (1969) and by Spotnitz et al. (1966). Although these data have been obtained for experiments on dog hearts, we will apply them to the rabbit hearts which were used in our experiments. Our aim here is merely to test the model sensitivity in a relevant range of parameter values. The situation at a preload of $0 \mathrm{mmHg}$ is called the reference situation or undeformed state.

For calculating the ventricular pressure during the active phase of contraction, the following assumptions will be made:

(1) The parameters of all muscle fibres are the same and the fibres are incompressible.

(2) All muscle fibres contract synchronously. This assumption is made because the time course of the spread of excitation over the ventricle is not exactly known. Besides, a non synchronous excitation of all muscle fibres rather complicates the analysis. For these reasons in the experiments all muscle fibres were stimulated synchronously using large sheet electrodes. It means also that probably hardly any torsion and isovolumic shape changes of the ventricle will be present (Arts, 1978). See also assumption 3 and 4.

(3) Volume changes will take place in such a way that a point $\left(r_{0}, \theta_{0}, z_{0}\right)$ in the wall of the undeformed cylinder with length $L_{0}$ and volume $V_{0}$, transposes to the point $(r, \theta, z)$ of the deformed cylinder with length $L$ and volume $V$, according to

$\theta=\theta_{0} ; \quad z=e_{z} z_{0} ; \quad r=\left[\left(r_{0}^{2}+\frac{V-\nu_{0}}{\pi L_{0}}\right) / e_{z}\right]^{1 / 2}$,

where $e_{z}$ represents the relative length of the cylinder $\left(L / L_{0}\right)$.

(4) The relative cylinder length $e_{z}$ will depend on ventricular volume. As a first approximation, a linear relation is used which suffices for estimating sign and order of magnitude of shape changes

$$
e_{z}=1+\frac{V_{p}-V_{0}}{V_{0}} e_{p}+\frac{V-V_{p}}{V_{0}} e_{e} .
$$

The constants $e_{p}$ and $e_{e}$ determine these shape changes resulting from a preload change and during ejection, respectively. During isovolumic contraction $\left(V=V_{p}\right)$, $e_{x}$ is constant. During ejection $[V=V(t)], e_{z}$ changes continuously.

(5) Due to the assumed deformation (equation 10) and the assumption that during the active phase there is no interaction between muscle fibres in locally parallel planes (Wong, 1973), it follows that bending moments are not present in the wall. The only stresses will be tensile stresses generated by the sarcomeres and the isotropic or hydrostatic pressure $p(r)$. Furthermore, wall acceleration will have no influence on the generated pressure (Tallarida et al., 1970; Back, 1977) and pressure gradients in the ventricle during ejection will be neglected (Gordon, 1976; Back, 1977).

The pressure in the cylinder wall is determined by 
the mechanical equilibrium equation in the r-direction

$$
\frac{1}{r} \frac{\partial\left[r \sigma_{r r}(r)\right]}{\partial r}-\frac{\sigma_{\theta \theta}(r)}{r}=0,
$$

where $\sigma_{r r}(r)$ and $\sigma_{\theta \theta}(r)$ are the principal stresses in the $r$ and $\theta$ direction, respectively. From assumption (5) follows

$$
\begin{aligned}
& \sigma_{r r r}(r)=-p(r) \\
& \sigma_{\theta \theta}(r)=-p(r)+\sigma_{m, \theta \theta}(r) .
\end{aligned}
$$

$\sigma_{m .0 t}$ is the stress generated by the muscle fibre. Since the fibre makes an angle $\phi(r)$ with the $(r, \theta)$ plane, we finally get

$$
\sigma_{m . \theta \theta}(r)=\frac{1}{V M} F_{m}(r) l_{m}(r) \cos ^{2} \phi(r),
$$

where $V M$ is the volume of the fibre.

Together with the equations (12) and (13), the pressure over the wall, i.e. the differences in pressure at the inner and outer radius $R_{1}$ and $R_{2}, P=p\left(R_{1}\right)-p\left(R_{2}\right)$ becomes

$$
P=\frac{1}{V M} \int_{R_{1}}^{R_{2}} F_{m}(r) l_{m}(r) \cos ^{2} \phi(r) \frac{\mathrm{d} r}{r} .
$$

In the experimental situation, the inner volume $V$ and the wall volume $V_{w}$ can be estimated, so $R_{1}$ and $R_{2}$ will be expressed in these quantities

$$
R_{1}=\left(\frac{V}{\pi L}\right)^{1 / 2} ; \quad R_{2}=\left(\frac{V+V_{w}}{\pi L}\right)^{1 / 2} .
$$

For the solution of equation (15) at different preloads and during ejection see Appendix.

From the dimensional analysis it follows that the pressure difference $P$ does not depend on both $R_{1}$ and $R_{2}$, but only on their ratio. The ratio $R_{1} / R_{2}$ does not contain ventricular length, so this length does not need to be specified.

\section{EXPERIMENTAL METHODS}

The experimental set up and the heart preparation has been previously described by Boom et al. (1973) and Nieuwenhuijs (1976). The apparatus was modified in such a way that nonisovolumic experiments could also be performed. To satisfy condition (2) (see Ventricular model), the isolated rabbit hearts from which the left atria were removed were stimulated by large sheet electrodes with current pulses of such a strength that no increase in developed pressure could be achieved by increasing stimulation current. The bearts were perfused via the aorta with a constant pressure of $50 \mathrm{~mm} \mathrm{Hg}$. End-diastolic pressure could be controlled and adjusted via the mitral orifice. Ejection also took place via the mitral orifice. The afterload consists of a horizontal tube in which a counter valve and a flow probe were mounted, and a vertical tube filled with liquid to an adjustable beight (see Fig 2).

Intra ventricular pressure was measured using a catheter tip manometer (Millar PC 350). The flow out of the ventricle was recorded with an extracorporal electromagnetic flow probe (Scalar $\phi 3 \mathrm{~mm}$ ). These data, together with calibration and control signals, were sampled on-line $(1 \mathrm{kHz})$ by a computer and subsequently processed. The reference volume $V_{0}$ was determined by emptying the heart after the experiment. An estimation of $V_{w}$ was found by weighing the

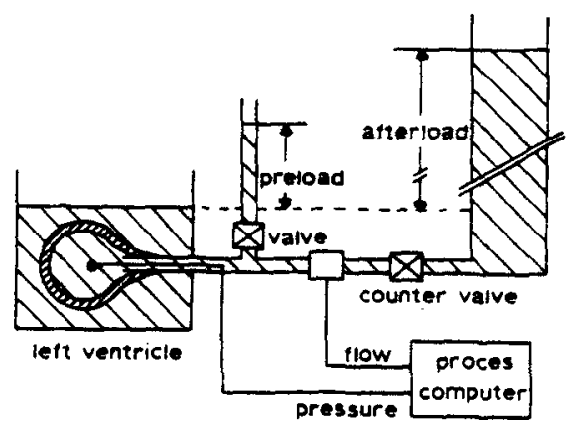

Fig. 2. Experimental set-up.

ventricle. The values for $V_{0}$ and $V_{w}$ obtained in this way may however be subjected to an error of about $20 \%$.

\section{RESULTS}

Figure 3(a) represents developed pressure recordings of a number of isovolumic contractions at different preloads. In Fig. 3(b) the curves have been normalised with respect to their maximum value. It is seen that the ascending limbs now more or less coincide, while the descending limbs become relatively steeper at lower preloads. In Fig. 4 an example is given of a set of isovolumic contractions at a preload of

(a)

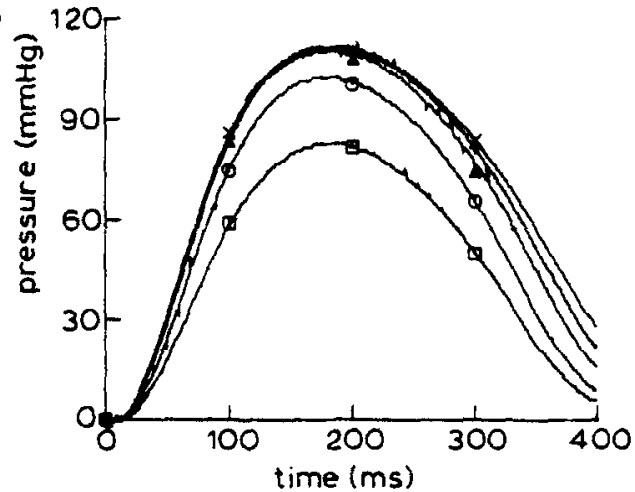

(b)

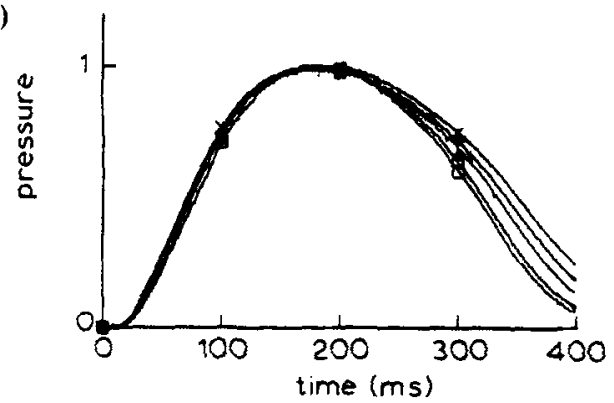

Fig. 3. Developed pressure recordings of isovolumic contractions at different preloads (a) not normalised (b) normalised. Preloads of $0,1,2,3$ and $4 \mathrm{mmHg}$ correspond with the symbols $(\square),(O),(\Delta),(+)$ and $(x)$, respectively. As a result of the analog-digital conversion, the tracings are not very smooth. 


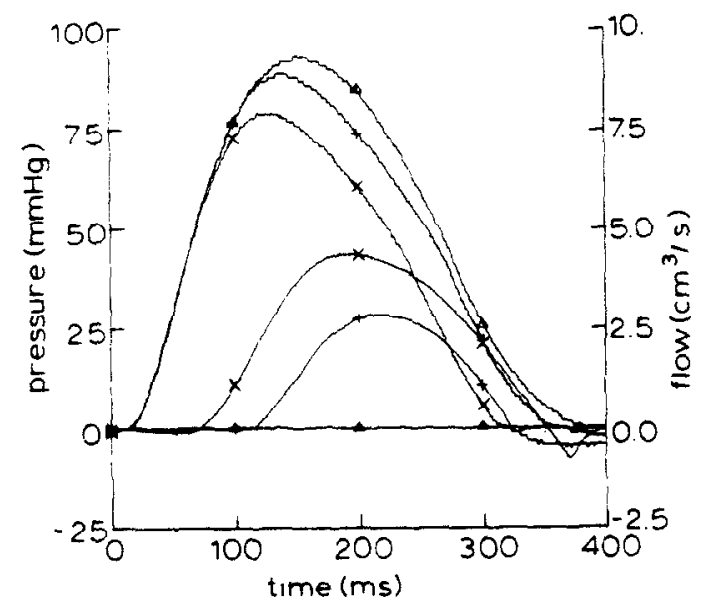

Fig. 4. Developed pressure recordings (upper 3 curves) and corresponding flow recordings (lower 3 curves) at a preload of $2 \mathrm{mmHg}$ and an afterload of $100 \mathrm{mmHg}(\triangle), 80 \mathrm{mmHg}(+)$ and $50 \mathrm{mmHg}(x)$.

$2 \mathrm{mmHg}$ and different afterloads. This figure shows that the contraction is shortened at lower afterloads. Because of the simple construction generating the afterload, the flow courses do not resemble those measured in vivo experiments. For our purposes, however, this is of no importance.

The model contains a great number of parameters. Some of them can be determined experimentally ( $V_{0}$, $V_{w}$ ), some are taken from the literature $\left[h, g_{2}\right.$ (Huxley, 1957; Julian, 1969), force-length relation (Pollack and Krueger, 1976), fibre orientation (Streeter et al., 1969), sarcomere length distribution (Spotnitz et al., 1966)]. The values of $\Delta x, \Delta t, N L, x_{a}$ and $x_{b}$ are chosen to give reliable numerical results (see Appendix). The remaining unknown parameters have to be estimated. We do realise that with enough free parameters a model fit is always obtainable. However, it will appear that some of the parameters are rather critical for fitting the experimental results.

\section{(a) Isovolumic contractions}

Model parameters of isovolumic contractions are estimated by minimizing the sum of the squares of the deviation between measured and simulated pressure. For this aim a numerical minimizing procedure developed by Hooke and Jeeves (1961) is applied. The procedure is based on a direct search method, for which no derivatives of the function are required. Since more than one minimum might exist, several starting values are used to find the absolute minimum. The procedure is stopped when the step size in any direction becomes less than $0.1 \%$ of the current value of the parameters. All points sampled during the first $450 \mathrm{msec}$ were used for each twitch that was fitted.

The parameters fitted in the reference situation are those determining coupling rate $A, b_{1}, b_{2}$, decoupling rate $g_{1}$ and series elastic stiffness $\alpha$. After each parameter variation the simulated pressure curve is multi- plied by a factor so that its maximum value agrees with the maximum of the measured pressure. This factor represents unknown multiplicative factors in the pressure, namely cross-bridge stiffness $Z$, fibre volume $V M$ and the maximum of the myosin head density $N(s) . F_{0}$ was initially chosen equal to 1 , a value not in contradiction to the literature, and varied afterwards. Table 1 shows the results. $t_{0}, P_{\max }$ and $t P_{\max }$ are directly estimated from pressure recordings. $\overline{\Delta P}$ equals the root mean square deviation between experiment and model fit. The maximum sarcomere shortening in the middle layer $\varepsilon_{\text {s.max }}$ gives an indication of the magnitude of the SEE. To investigate the infiuence of the SEE, the stiffness $\alpha$ is not estimated in situation 1, but a very high value is assumed, which results in an isometric sarcomere contraction. In situation $2, \alpha$ is fitted also. This gives a much better fit, since $\overline{\Delta P}$ is now about equal to the experimental error. In situations 2,3 and 4 , the value of $F_{0}$ is varied. The overall fit did not improve, but $g_{1}, \varepsilon_{s . \max }$ and $\alpha$ are clearly influenced. A low value of $F_{0}$ results in high sarcomere stretches at preload 0 , while a high value of $F_{0}$ gives rise to high stretches at greater preloads. $F_{0}=1$ will be chosen. The results of two other experiments are presented in 5 and 6. These three experiments suggest that $f_{\max }$ and $g_{1}$ are of the order of $150-200 \mathrm{sec}^{-1}$, while due to the SEE the maximum sarcomere shortening is about $6 \%$. Figure 5 shows a model fit with and without a SEE (situation 2 and 1, respectively), as well as the course of $f$ (situation 2).

Contractions at higher preloads are fitted by using the parameter set found in the reference situation (situations 2, 5 and 6 of Table 1) and by estimating new inner volume $V_{p}$ and the factor describing the influence of preload on the activation $b_{p}$ (see equation 9 ). Table 2 shows the results. The influence of $b_{p}$ is shown in the situations 1 and 2 and in 4 and 5 . When the activation function is not changed $\left(b_{p}=0\right)$, the results are clearly worse than when $b_{p}$ is fitted, especially at higher preloads. In 3 and 6 the length of the cylinder $e_{z}$ is changed by varying $e_{p}$ (see equation 11). No

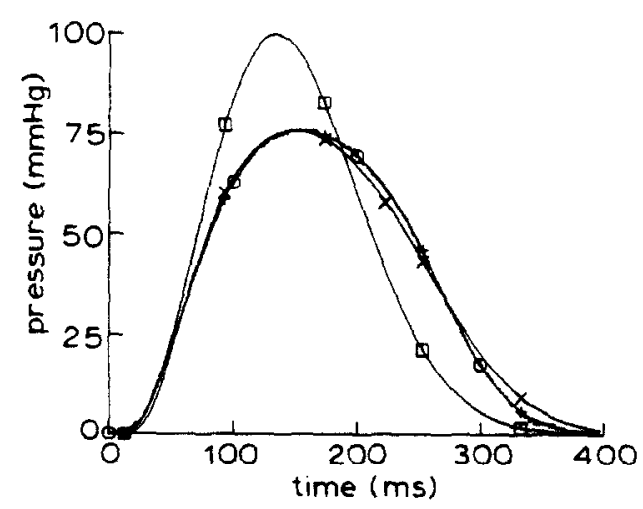

Fig. 5. Isovolumic contraction in the reference situation. Measured pressure $(O)$, best fit of simulated pressure with a SEE $(+)$, corresponding activation function $(\square)$, best fit of simulated pressure without a $\operatorname{SEE}(x)$ 


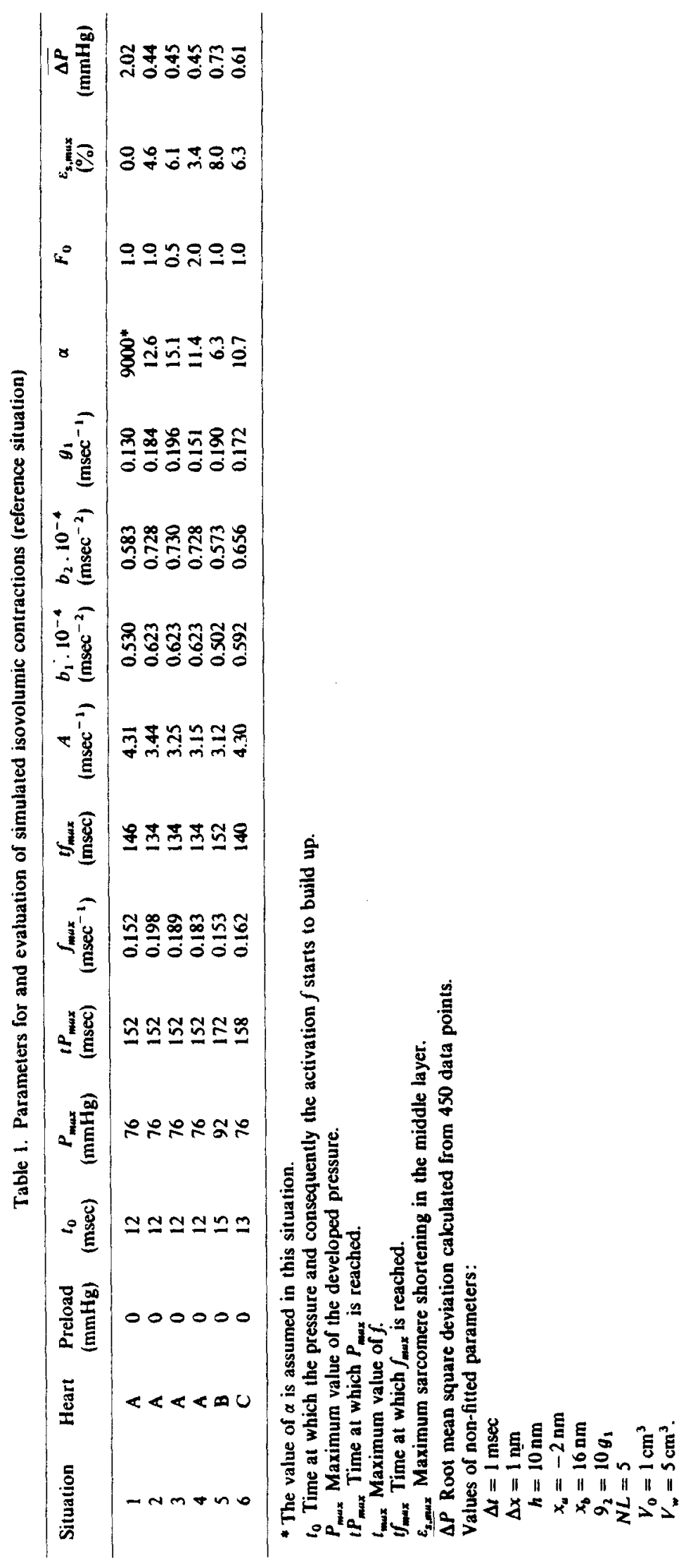


Table 2. Isovolumic contractions at higher preloads

\begin{tabular}{|c|c|c|c|c|c|c|c|c|c|}
\hline Situation & Heart & $\begin{array}{l}\text { Preload } \\
\text { (mmHg) }\end{array}$ & $\begin{array}{c}t_{0} \\
\text { (msec) }\end{array}$ & $\underset{\left(m m H_{g}\right)}{P_{\max }}$ & $\begin{array}{l}t P_{\max } \\
(\text { msec })\end{array}$ & $\begin{array}{c}V_{p^{3}} \\
\left(\mathrm{~cm}^{3}\right)\end{array}$ & $b_{p}$ & $e_{p}^{*}$ & $\begin{array}{c}\overrightarrow{\Delta P} \\
(\mathrm{mmHg})\end{array}$ \\
\hline 1 & A & 1 & 11 & 86 & 145 & 1.15 & $0.00^{*}$ & 0.0 & 0.90 \\
\hline 2 & A & 1 & 11 & 86 & 145 & 1.15 & 0.74 & 0.0 & 0.66 \\
\hline 3 & A & 1 & 11 & 86 & 145 & 1.19 & 0.57 & 0.2 & 0.66 \\
\hline 4 & A & 2 & 10 & 93 & 149 & 1.30 & $0.00^{*}$ & 0.0 & 2.34 \\
\hline 5 & A & 2 & 10 & 93 & 149 & 1.27 & 0.86 & 0.0 & 0.99 \\
\hline 6 & A & 2 & 10 & 93 & 149 & 1.37 & 0.61 & 0.2 & 1.01 \\
\hline 7 & B & 1 & 11 & 110 & 164 & 1.33 & 0.98 & 0.2 & 1.10 \\
\hline 8 & $\mathbf{B}$ & 2 & 8 & 120 & 169 & 1.53 & 0.89 & 0.2 & 0.78 \\
\hline 9 & $C$ & 1 & 9 & 79 & 141 & 1.07 & 2.94 & 0.2 & 0.77 \\
\hline 10 & C & 2 & 9 & 89 & 149 & 1.29 & 0.94 & 0.2 & 1.52 \\
\hline
\end{tabular}

${ }^{*} b_{p}$ is not fitted in this situation, neither are the values of $e_{p}$.

improvement is found. In the literature no accurate data about shape changes at different preloads are available. From the statical equilibrium between the pressure acting on the upper plane of the cylinder and the wall stress in the z-direction, it follows that at higher preloads the inner radius has to increase relatively more than the length. To achieve this, a value of $e_{p}=0.2$ is chosen. The situations $7-10$ represent the results of the two other experiments. $b_{p}$ is reasonably constant, particularly in the hearts $A$ and $B$, so that equation (9) seems to give a rather good description of the influence of preload on the activation function. Figure 6 gives the measured and calculated isovolumic pressure courses of heart $A$ at preloads of 0,1 and $2 \mathrm{mmHg}$ with preload dependent values of $b_{1}$ and $e_{p}=$ 0.2 .

\section{(b) Non-isonolumic contractions}

Pressure changes during non-isovolumic contractions are simulated using the measured flow and the parameters describing isovolumic contraction. At zero preload these simulations are done for several choices of some of the non-fitted parameters (see Table 3). In situation 1 the parameters from situation 2, Table 1 , are used, together with the assumptions that there is no influence of ejection on the activation $\left(b_{e}=0\right)$ and on

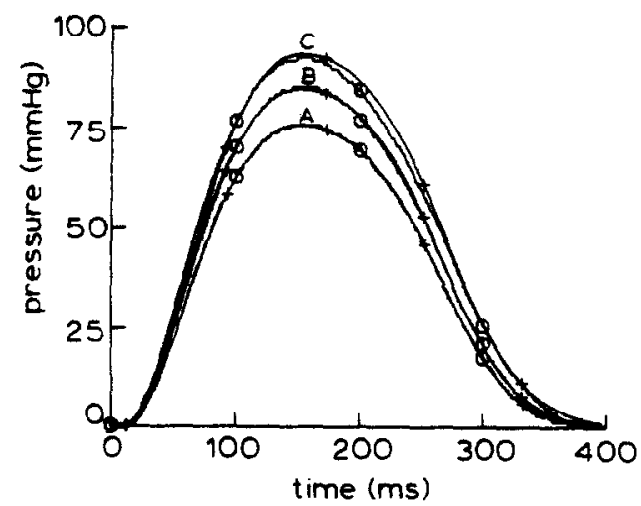

Fig. 6. Isovolumic contractions at different preloads. From $A$ to $\mathrm{C}$ the preload increases from 0 to $2 \mathrm{mmHg}$. Measured pressure $(O)$, best fitted simulated pressure $(+)$.
Table 3. Influence of some parameter variations during a non-isovolumic contraction at a preload of $0 \mathrm{mmHg}^{-}$

\begin{tabular}{ccc}
\hline Situation & $\begin{array}{c}\text { Modification with regard } \\
\text { to situation } 1\end{array}$ & $\begin{array}{c}\overline{\Delta P} \\
(\mathrm{mmHg})\end{array}$ \\
\hline 1 & - & 3.3 \\
2 & $\alpha=9000$ & 5.7 \\
3 & $\phi\left(r_{0}\right)$ straight line from & \\
& $80^{\circ}$ at $R_{1}$ till $-80^{\circ}$ at $R_{2}$ & 2.7 \\
4 & $h=20 \mathrm{~nm}$ & 2.5 \\
5 & $V_{0}=1.25 \mathrm{~cm}^{3}$ & 2.4 \\
6 & $V_{\mathrm{w}}=8.00 \mathrm{~cm}^{3}$ & 2.3 \\
7 & $e_{e}=-0.1$ & 3.8 \\
8 & $e_{e}=0.1$ & 2.9 \\
9 & $b_{e}=0.3$ & 3.3 \\
\hline
\end{tabular}

In all cases: heart $A ; t_{0}=12 \mathrm{msec} ; P_{\max }=73 \mathrm{mmHg}$; $t P_{\max }=138 \mathrm{msec} ; Q_{\max }$ (maximum value of the flow) $=$ $2.0 \mathrm{~cm}^{3} / \mathrm{sec} ; t Q_{\max }$ (time at which $Q_{\max }$ is reached) $=$ $205 \mathrm{msec}$. The ejection fraction calculated from the measured flow, is in all cases $25 \%$, except situation 5 where it is $20 \%$ because a higher inner volume is assumed.

the cylinder length $\left(e_{e}=0\right)$. In the other situations, one parameter is always modified with regard to situation 1. In 2 a very stiff SEE is assumed (see situation 1 of Table 1), which clearly leads to a worse result. For a very stiff SEE, muscle shortening velocity $v_{m}$ (which is determined by the flow) equals sarcomere shortening velocity $v_{s}$ (see equation 3 ). When the SEE is less stiff, a part of $v_{m}$ is taken over by $v_{e}$ during pressure relaxation, so that the influence of the flow on the muscle force and also on the pressure becomes smaller. Situation 1 and 2 are plotted in Fig. 7. An increase in sarcomere orientation (situation 3) gives a somewhat better result. An increase in maximum cross-bridge length (situation 4) leads to a diminishing of the influence of $v_{s}$ on $F_{s}$. An increase in inner volume and wall volume (situation 5 and 6 ) results in a decrease of $v_{m}$ at the same flow. Lengthening of the cylinder during flow (negative value of $e_{e}$, situation 7) has an effect in the wrong direction, while shortening (situation 8) leads to better results. This finding of shortening of ventricular length during ejection is in agreement with measurements made by Ross et al. (1967) and Rankin et al. (1976). Since during flow the contraction is 


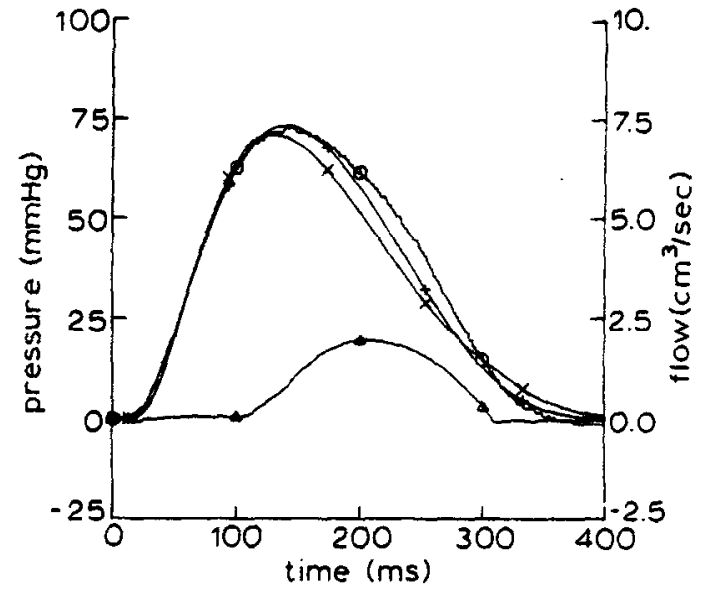

Fig 7. Non-isovolumic contraction in the reference situation. Measured pressure $(O)$, measured flow $(\Delta)$, calculated pressure with a SEE $(+)$, calculated pressure without a $\operatorname{SEE}(x)$.

shortened (see Fig. 4), a positive value of $b_{e}$ is chosen (situation 9). However, the effect is negligible.

Simulations with flow at preloads of 1 and $2 \mathrm{mmHg}$ are given in Table 4. The parameters used are obtained from Table 2 for $e_{\mathrm{p}}=0.2$. During flow $e_{e}=0.1$ is assumed. From situation 2 and 3 and from 4 and 5 it follows that variation of $b$, has no clear influence on $\overline{\Delta P}$. Equation (9) seems to be not very appropriate to describe the phenomena of shortening of contraction at lower afterloads. Henceforth $b_{e}=0.0$ will be chosen. Figure 8 represents situation 1 and 2 , together with the isovolumic contraction of situation 3, Table 2. Almost all the other cases of Table 4 give the same figure. In the first part of the ejection the calculated pressure exceeds the measured one, while in the middle part it is lower and at the end of the relaxation it is higher. Since due to ejection ventricular volume has been decreased, the measured pressure drops a few millimeters of mercury below the initial end-diastolic pressure at the end of the relaxation. As a result of this effect, caused by the passive pressure-volume relation, a relatively larger error may occur (Fig. 8), particularly at the end of the contraction. A root mean square deviation of at least $2-3 \mathrm{mmHg}$ may be expected.

\section{DISCUSSION}

The aim of this study was to develop a model for left ventricular contractions based as much as possible on current physiological knowledge. This approach leads in general to relatively complex models with a great number of parameters. With enough free parameters the model may be fitted to almost any result. For this reason the number of parameters should be kept as low as possible.

In recent muscle models (Huxley and Simmonds, 1971 ; Julian et al., 1974) it is supposed that crossbridge formation is a two stage process. Such models were developed to describe rather fast phenomena. Since in normal contracting hearts sarcomere shortening is not that fast, our model is based upon Huxley's original concept. [In literature there is some discussion

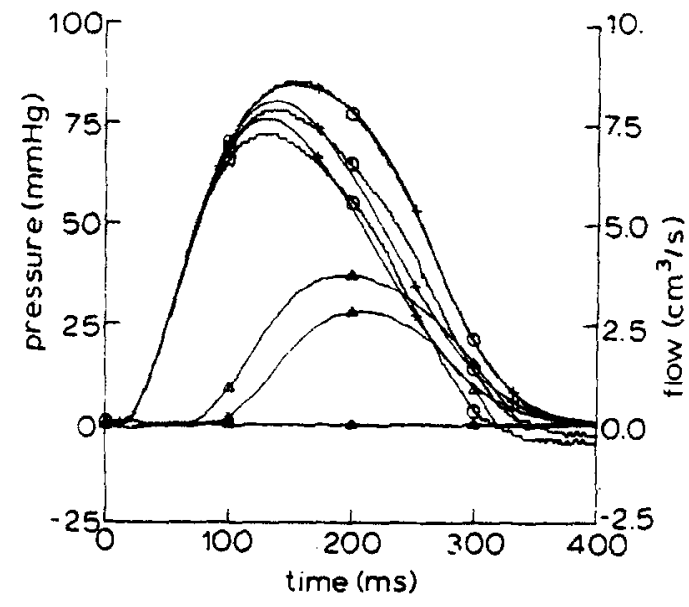

Fig. 8. Non-isovolumic contractions at a preload of $1 \mathrm{mmHg}$ and different afterloads. Measured pressure $(O)$, measured flow $(\Delta)$, calculated pressure $(+)$.

Table 4. Results of calculations on non-isovolumic contractions

\begin{tabular}{|c|c|c|c|c|c|c|c|c|c|c|}
\hline Situation & Heart & $\begin{array}{l}\text { Preload } \\
\text { (mmHg) }\end{array}$ & $\begin{array}{c}t_{0} \\
\text { (msec) }\end{array}$ & $\underset{(\mathrm{mmH}}{P_{\max }}$ & $\begin{array}{c}t P_{\max } \\
(\mathrm{msec})\end{array}$ & $\begin{array}{c}Q_{\max } \\
\left(\mathrm{cm}^{3} / \mathrm{sec}\right)\end{array}$ & $\begin{array}{l}t Q_{\max } \\
(\mathrm{msec})\end{array}$ & $\begin{array}{c}\text { Ejection } \\
\text { fraction } \\
(\%) \\
\end{array}$ & $b_{e}$ & $\begin{array}{c}\overline{\Delta P} \\
(\mathrm{mmHg})\end{array}$ \\
\hline 1 & $\mathbf{A}$ & 1 & 11 & 78 & 126 & 2.8 & 206 & 34 & 0.0 & 2.8 \\
\hline 2 & $\ddot{A}$ & 1 & 11 & 72 & 122 & 3.7 & 190 & 52 & 0.0 & 4.1 \\
\hline 3 & A & 1 & 11 & 72 & 122 & 3.7 & 190 & 52 & 0.3 & 3.8 \\
\hline 4 & A & 2 & 10 & 89 & 134 & 2.9 & 215 & 28 & 0.0 & 3.4 \\
\hline 5 & A & 2 & 10 & 89 & 134 & 2.9 & 215 & 28 & 0.3 & 4.0 \\
\hline 6 & A & 2 & 10 & 79 & 119 & 4.4 & 195 & 54 & 0.0 & 4.1 \\
\hline 7 & B & 1 & 11 & 107 & 168 & 2.0 & 241 & 19 & 0.0 & 3.9 \\
\hline 8 & $\mathbf{B}$ & $i$ & 11 & 86 & 158 & 4.0 & 184 & 62 & 0.0 & 7.4 \\
\hline 9 & B & 2 & 8 & 111 & 139 & 3.1 & 237 & 32 & 0.0 & 6.3 \\
\hline 10 & B & 2 & 8 & 99 & 131 & 4.4 & 189 & 59 & 0.0 & 7.5 \\
\hline 11 & C & 1 & 9 & 78 & 146 & 1.5 & 192 & 15 & 0.0 & 4.2 \\
\hline 12 & C & 1 & 9 & 76 & 162 & 2.8 & 154 & 33 & 0.0 & 7.5 \\
\hline 13 & $\mathrm{C}$ & 2 & 9 & 84 & 127 & 1.7 & 193 & 15 & 0.0 & 3.5 \\
\hline 14 & C & 2 & 9 & 77 & 161 & 3.1 & 160 & 33 & 0.0 & 5.8 \\
\hline
\end{tabular}

The values of $b$, are assumed in all situations. 
about the cross-bridge theory (Noble and Pollack, 1977). However, this theory still remains workable and generally accepted]

The ventricular geometry is approximated by a cylinder, which is an improvement with regard to spherical models (Back, 1977), particularly since sarcomere orientation can be implemented and the influence of shape changes can be investigated. A cylindrical model can give a good approximation of wall stress in the equatorial region of the left ventricle. To describe wall stress in the apical region, an ellipsoidal model would be more appropriate (Back, 1977). The equatorial region is however, more representative than the apical region. Our preliminary findings (Van den Broek and Denier van der Gon, 1979) suggest that a more complicated thick-walled truncated ellipsoidal model containing more parameters is only useful if ventricular inner and outer dimensions can be measured accurately.

The model was fitted to data obtained from experiments. The aim was merely to obtain an order of magnitude of the model parameters. For this purpose the rather non-physiological stimulation and afterload was suitable. The sensitivity of the model was tested using these estimated values as starting values. From the results obtained we can conclude:

(a) A kind of series elastic element appears to be essential, particularly for the description of nonisovolumic contractions. For isovolumic contractions, maximum sarcomere stretches of about $6 \%$ are found. These values do not seem to be unrealistic, since during contraction of the rather complex structure of the left ventricle, all kinds of effects resulting in sliding may occur. These effects are not explicitly incorporated in the model, but they are lumped together in the series elasticity. In experiments on papillary muscle even higher sarcomere stretches are often found, which are however, mostly caused by the damaged ends of the muscles (Pollack and Krueger, 1976).

(b) The chosen activation function allows fits with an accuracy of $0.5 \mathrm{mmHg}$. This lies within the measurement error range. The shape of $f$ is rather symmetrical and its maximum lies near the maximum of the isovolumic pressure (see Fig. 5). These findings agree with direct measurements of calcium transients in muscle cells (Blinks, 1973). In the way described in this study (equation 9), the activation function $f$ appears to be preload dependent, while the influence of ejection on $f$ is not very critical. The order of magnitude of $f$ and $g$ agrees with values which are often suggested in muscle models.

(c) Ventricular dimensions $\left(V_{0}, V_{w}\right)$ are important parameters. The influence on $\overline{\Delta P}$ of a shape change due to preload variation is small, but the model fit is rather sensitive to a shape change during ejection. In practise it will thus be necessary to measure simultaneously with pressure and flow the inner and outer dimensions of the ventricle. In this way the parameters $V_{0}, V_{w}, V, e_{p}$ and $e_{e}$ can be determined to reduce the number of assumptions and simplify the curve-fitting procedures.
More reliable estimates of the remaining parameters may be obtained.

(d) Adding of a parallel elastic element to the model enables a better fit in particular at the end of a nonisovolumic contraction. For that purpose, passive pressure-volume relations have to be known, or should be estimated from the recordings also.

Acknowledgements - The authors wish to thank J. H. M. Nieuwenhuijs and $P$. Schiereck for their cooperation with the experiments and A. Crowe, R. M. Heethaar and E. A. Wolfs for their helpful criticism of this manuscript.

\section{REFERENCES}

Allen, D. G., Jewell, B. R. and Murray, J. W. (1974) The contribution of activation processes to the length-tension relation of cardiac muscle. Nature. Lond. 248, 606-607.

Arts, M. G. J. (1978) A mathematical model of the dynamics of the left ventricle and the coronary circulation. Thesis. State University of Limburg.

Back, L. H. (1977) Left ventricular wall and fluid dynamics of cardiac contraction. Mathl. Biosc. 36, 257-297.

Blinks, J. R. (1973) Calcium transients in striated muscle cells. Eur. J. Cardiol. 1/2, 135-142.

Boom, H. B. K., Denier van der Gon, J. J., Nieuwenhuijs, J. H. M. and Schiereck, P. (1973) Cardiac contractility: actin-myosin interaction as measured from the left ventricular pressure curve. Eur. J. Cardiol. 1/2, 217-224.

Gordon, D. G. (1976) The physics of left ventricular ejection and its implications for muscle mechanics. Eur. J. Cardiol. 4, Suppl., 87-95.

Grood, E. S. and Mates, R. E. (1975) Intluence of cross-bridge compliance on the force-velocity relation of muscle. Am.J. Physiol. 228, 244-249.

Hooke, R. and Jeeves, T. A. (1961) Direct search solution of numerical and statistical problems. J. Ass. Comp. Mach. 8, 212-229.

Huxley, A. F. (1957) Muscle structure and theories of contraction. Prog. Biophys. biophys. Chem. 7, 255-318.

Huxley, A. F. and Simmonds, R. M. (1971) Proposed mechanism of force generation in striated muscle. Nature, Lond. 233, 533-538.

Jewell, B. R. (1977) A re-examination of the influence of muscle length on myocardial performance. Circulation Res. 40, $221-230$.

Julian, F. J. (1969) Activation in a skeletal muscle contraction model with a modification for insect fibrillar muscle. Biophys. J. 9, 547-570.

Julian, F. J., Sollins, K R. and Sollins, M. R. (1974) A model for the transient and steady-state mechanical behaviour of contracting muscle. Biophys. J. 14, 546-562.

Kaufmann, R., Bayer, R., Fürniss, T., Krause, $H$. and Tritthart, H. (1974) Calcium-movement controlling cardiac contractility- $\Pi$. Analog computation of cardiac excitation-contraction coupling on the basis of calcium kinetics in a multi-compartment model. $J$. molec. cell. Cardiol. 6, 543-559.

Lakatta, E. G. and Jewell, B. R. (1977) Length dependent activation. Its effect on the length-tension relation in cat ventricular muscie. Circulation Res. 40, 251-257.

Nieuwenhuijs, J. H. M. (1976) Kontraktiliteit van de linker ventrikel. Thesis, State University of Utrecht.

Noble, M. I. M. (1977) The diastolic viscous properties of cat papillary muscle. Circulation Res. 40, 288-292.

Noble, M. I. M. and Pollack, G. H. (1977) Molecular mechanism of contraction. Circulation Res. 40, 333-342.

Pollack, G. H. and Krueger, J. W. (1976) Sarcomere dynamics in intact cardiac muscle. Eur. J. Cardiol. 4, Suppl., 53-65.

Rankin, J. S., Machall, P. A., Arentzen, C. E., Ling, D., Greenfield, J. C. and Anderson, R. W. (1976) The three- 
dimensional dynamic geometry of the left ventricle in the conscious dog. Circulation Res. 39, 304-313.

Ross, J. Jr., Sonnenblick, E. H., Covell, J. W., Kaiser, G. A. and Spiro, D. (1967) The architecture of the heart in systole and diastole. Circulation Res. 21, 409-421.

Ross, M. A. and Streeter, D. D., Jr. (1975) Nonuniform subendocardial fibre orientation in the normal macaque left ventricle. Eur. J. Cardiol 3/3, 229-247.

Schiereck, P. and Boom, H. B. K. (1978) Left ventricular active stiffness: dependency on time and inotropic state. Pflügers Arch. ges. Physiol. 374, 135-143.

Smith, G. D. (1965) Numerical Solutions of Partial Differential Equations. Oxford University Press, London.

Spotnitz, H. M., Sonnenblick, E. H. and Spiro, D. (1966) Relation of ultrastructure to function in the intact heart: sarcomere structure relative to pressure-volume curves of intact left ventricles of dog and cat. Circulation Res. 18, 49-66.

Stein, R. B. and Wong, E. Y. M. (1974) Analysis of models for the activation and contraction of muscle. J. theoret. Biol. 46, 307-327.

Stetter, H. J. (1961) On the convergence of characteristic finite-difference methods of high accuracy of quasi-linear hyperbolic equations. Num. Math. 3, 321-344.

Streeter, D. D., Spotnitz, H. M., Patel, D. P., Ross, J., Jr. and Sonnenblick, E. H. (1969) Fibre orientation in the canine left ventricle during diastole and systole. Circulation Res. 24, 339-347.

Streeter, D. D., Jr., Vaishnav, R. N., Patel, D. J., Spotnitz, J. M., Ross, J., Jr. and Sonnenblick, E. H. (1970) Stress distribution in the canine left ventricle during diastole and systole. Biophys. J. 10, 345-363.

Tallarida, R. J., Rusy, B. F. and Loughnane, M. H. (1970) Left ventricular wall acceleration and the law of Laplace. Cardiovasc. Res. 4, 217-223.

Van den Broek, J. H. J. M. and Denier van der Gon, J. J. (1979) A model for left ventricular contractions based on the sliding filament theory. Cardiac Dynamics (Edited by Baan, J., Arntzenius, A. C. and Yellin, E. L.) Martinus Nijhoff. The Hague.

Weber, A. and Murray, J. M. (1973) Molecular control mechanisms in muscle contractions. Physiol. Rev. 53, 612-673.

Wong, A. Y.K. (1971) Mechanics of cardiac muscle, based on Huxley's model: mathematical simulation of isometric contraction. J. Biomechanics 4, 529-540.

Wong, A. Y. K. (1973) Myocardial mechanics : application of sliding-filament theory to isovolumic concentration of the left ventricle. J. Biomechanics 6, 565-581.

Yoran, C., Covell, J. W. and Ross, J., Jr. (1973) Structural basis for the ascending limb of left ventricular function. Circulation Res. 32, 297-303.

\section{NOMENCLATURE}

$l_{s} v_{s} F_{g}$
$l_{p} v_{e} F_{e}$
$l_{m p} v_{m}, F_{m}$
$l_{0}$
$F_{0,} \alpha$
$R_{1}, R_{2}$
$V_{0}$
$V_{p}$
$V$
$V_{w}$
$N L$
$e_{z}$
$e_{p} e_{e}$
$s$
$h$
$t$

length, velocity and force of the half-sarcomere length, velocity and force of the SEE length, velocity and force of the muscle fibre muscle length at zero preload dimensionless parameters of the SEE inner and outer radius of the cylinder volume at zero filling pressure volume at higher preload volume during ejection wall volume number of wall layers relative length of the cylinder parameters describing the influence of preload and ejection on $e_{z}$, respectively sarcomere length maximum bridge length for attachment time

\author{
$t_{0}$ \\ $\boldsymbol{x}$ \\ $n(x, t)$ \\ $N(s)$ \\ $f(t)$ \\ $g_{1}, g_{2}$ \\ $A, b_{1}, b_{2}$ \\ $b_{0}$ \\ $b_{p} b_{\text {e }}$ \\ time at which the contraction starts \\ cross-bridge length \\ density of coupled bridges \\ density of maximum possible bridges \\ rate constant for making a cross-bridge \\ rate constants for breaking a cross-bridge \\ parameters of $f$ \\ value of $\dot{b}_{1}$ in the reference situation \\ parameters describing the influence of preload \\ and ejection on $b_{1}$, respectively.
}

\section{APPENDIX}

\section{Numerical Methods}

The integral for the pressure in the ventricle (15) is approximated by a sum over a number of NL equidistant wall layers. In each layer the value of the integrand is taken in the middle of that layer. From the simulations, 5 layers seem to be sufficient to estimate the integral with a $1 \%$ accuracy.

The functions $\phi(r)$ and $l_{m}(r)$ in the deformed state can be calculated from the given values $\phi\left(r_{0}\right)$ and $l_{m}\left(r_{0}\right)$ in the undeformed state. Consider an arbitrary point $\left(r_{0}, \theta_{0}, z_{0}\right)$ and a point one half-sarcomere down the helical path the muscle fibre makes (see Fig. 9). The coordinates of this last point are $\left(r_{0}, \theta_{p}, z_{p}\right)$. After deformation, the coordinates of the points become, respectively $\left(r, \theta_{0}, e_{2} z_{0}\right)$ and $\left(r, \theta_{p}, e_{2} z_{p}\right)$, according to condition 3 (ventricular model). From Fig. 9 follows

$$
\left.\left.\begin{array}{l}
\tan \phi\left(r_{0}\right)=\frac{z_{0}-z_{p}}{r_{0}\left(\theta_{0}-\theta_{p}\right)} \\
\tan \phi(r)=\frac{e_{z}\left(z_{0}-z_{p}\right)}{r\left(\theta_{0}-\theta_{p}\right)}
\end{array}\right\} \quad \begin{array}{r}
\phi(r)=\arctan \left[e_{z} \frac{r_{0}}{r} \tan \phi\left(r_{0}\right)\right] \\
\cos \phi\left(r_{0}\right)=\frac{r_{0}\left(\theta_{0}-\theta_{p}\right)}{l_{m}\left(r_{0}\right)} \\
\cos \phi(r)=\frac{r\left(\theta_{0}-\theta_{p}\right)}{l_{m}(r)}
\end{array}\right\} \quad \begin{aligned}
& I_{m}(r)=\frac{r}{r_{0}} \frac{\cos \phi\left(r_{0}\right)}{\cos \phi(r)} l_{m}\left(r_{0}\right) \\
& \text { (A2) }
\end{aligned}
$$

In the numerical calculations, discrete values of time $\Delta t$ are used. The volume $V(t+\Delta t)$ at a new point of time can be expressed during ejection in terms of $V(t)$ and the flow $Q$ out of the ventricle

$$
V(t+\Delta t)=V(t)-Q(t+\Delta t / 2) \Delta t .
$$

With the help of equations (10), (A1) and (A2), $\phi[r(t+\Delta t)]$ and $I_{m}[r(t+\Delta t)]$ can now be calculated. An approximation of the contraction velocity of the muscle fibre during $\Delta t$ can be given by

$v_{m}[r(t+\Delta t / 2)]=\left\{L_{m}[r(t+\Delta t)]-I_{m}[r(t)]\right\} / \Delta t$.

This velocity is necessary to compute the muscle force $F_{m}$ $[r(t+\Delta t)]$ in the various wall layers.

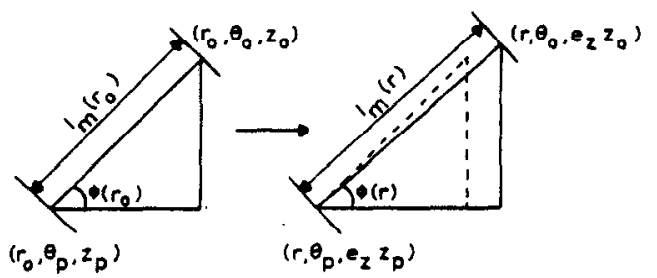

Fig. 9. Deformation of a part of an unfolded cylinder plane. 
The integral of equation (4) will be approximated by a sum between the boundaries $x_{a}$ and $x_{b}$, using the trapezoid rule. The boundaries are chosen in such a way that the contributions over the intervals $\left(-\infty, x_{a}\right)$ and $\left(x_{b}, \infty\right)$ can be neglected. With the definition of $x_{a}$ and $x_{b}$ the initial and boundary condition of equation (3) becomes

$$
\begin{array}{rrrr}
t \leqslant 0 & & n(x, t)=0 \\
t_{s} \leqslant 0 & \text { and } x>x_{b} & n(x, t)=0 \\
t_{s}>0 & \text { and } x<x_{a} & n(x, t)=0 .
\end{array}
$$

In each wall layer equation (3) has to be solved. Julian (1969) described a straightforward method (which is also used by Wong, 1971, Stein and Wong, 1974, and Grood and Mates, 1975). The method we will apply makes use of the characteristics of the equation which leads in general to more stable and accurate solutions.

The following equation holds along a line $l$ with slope $\lambda^{-1}$

$$
\left(\frac{\delta n}{\delta t}\right)_{1}=\frac{\partial n}{\partial t}+\lambda \frac{\partial n}{\partial x}
$$

From equation (3) we get

$$
\left(\frac{\partial n}{\partial x}\right)\left(v_{s}-\lambda\right)=q-p n-\left(\frac{\delta n}{\delta t}\right)_{1},
$$

where $q(s, x, t)=N(s) f(s, x, t)$ and $p(s, x, t)=f(s, x, t)+$ $g(s, x, t)$.

If $\lambda=v_{s}$ is chosen, equation (A7) is reduced to an equation which contains a total differential only

$$
\left(\frac{\delta n}{\delta t}\right)_{1}=q-p n
$$

$l$ is called the characteristic of the equation in the $x-t$ plane. To solve equation (A8) a rectangular grid is constructed with $a$ fixed mesh width $\Delta x$ in the $x$-direction and $a$, in principle variable width $\Delta t_{b}$, in the $t$-direction. The lines of the grid will be numbered $i$ and $j$, according to Fig. 10. The solution is convergent and stable under the condition

$$
\Delta t_{j} \leqslant\left|\frac{\Delta x}{v_{s}\left(t_{j}\right)}\right|
$$

(Smith, 1965). When an estimation of the maximum value of $v_{s}(t)$ can be made, it is possible to work with a fixed time step $\Delta t$, defined as

$$
\Delta t=\left|\frac{\Delta x}{v_{s, \max }}\right|
$$

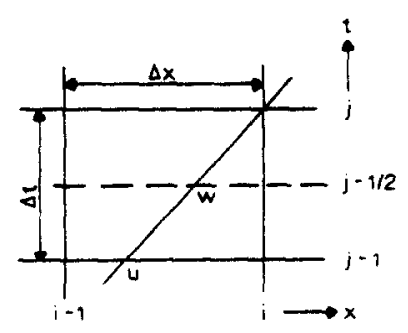

Fig. 10. The $x-t$ plane.

To calculate the value of $n$ at line $(j)$ from the value at line $(j-$ 1), we will apply a central-difference approximation round line $\left(j-\frac{1}{2}\right)$, which leads to an accuracy of order $\Delta x^{2}$ (Stetter. 1961). From point $(i, j)$ a characteristic is drawn with slope $v_{1, j-1 / 2}^{-1}$. The characteristic will cut line $\left(j-\frac{1}{2}\right)$ at point $w$ and line $(j-1)$ at point $u$ (see Fig. 10). $n(i, j)$ can now be expressed in $n(u)$ and the values of the coefficients in point $w$

$$
\frac{n(i, j)-n(u)}{\Delta t}=q(w)-p(w) \frac{n(i, j)+n(u)}{2},
$$

so

$$
\begin{array}{r}
n(i, j)=\frac{2-p(w) \Delta t}{2+p(w) \Delta t}\left[\frac{2 \Delta t q(w)}{2-p(w) \Delta t}+n(u)\right] . \\
=C_{1}\left[C_{2}+n(u)\right] .
\end{array}
$$

$n(u)$ can be expressed in $n(i-1, j-1)$ and $n(i, j-1)$; with

$$
c_{3}=v_{s, j-1 / 2} \frac{\Delta t}{\Delta x}
$$

and the boundary conditions (A5) we finally get

$$
\begin{aligned}
n(i, j)=c_{1}\left[c_{2}\right. & +c_{3} n(i-1, j-1) \\
& \left.+\left(1-c_{3}\right) n(i, j-1)\right] \text { for } v_{s . j-1 / 2} \geqslant 0 \\
n(i, j)=c_{1}\left[c_{2}\right. & +c_{3} n(i+1, j-1) \\
& \left.+\left(1-c_{3}\right) n(i, j-1)\right] \text { for } v_{2, j-1 / 2}<0 .
\end{aligned}
$$

So if $v_{z, j}$ is given, $n$ at time $t$, can be calculated. In general, $v_{2, j}$ is unknown, since it depends on the load of the muscle fibre. $v_{s, j}$ will be estimated so that $F_{s, j}$ equals $F_{e, r}$. This means that the zero of the function $G\left(v_{s, j}\right)=F_{s, j}-F_{e, j}$ has to be found. This is done numerically with a secant-method. $F_{3, j}$ follows from $n$ at line $j . F_{, j}$ can be calculated from $v_{s, j-1 / 2}$ and $v_{m, j-1 / 2}$ (given by equation A4)

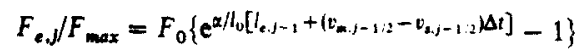

